\title{
A BIJECTIVE PROOF OF $r=1$ REDUCTION FORMULA FOR LITTLEWOOD-RICHARDSON COEFFICIENTS
}

\author{
Dong Ho MoON
}

\begin{abstract}
Inspired by the reduction formulae between intersection numbers on Grassmannians obtained by Griffiths-Harris and the factorization theorem of Littlewood-Richardson coefficients by King, Tollu and Toumazet, eight reduction formulae has been discovered by the author and others. In this paper, we prove $r=1$ reduction formula by constructing a bijective map between suitable sets of Littlewood-Richardson tableaux.
\end{abstract}

\section{Introduction}

We introduce basic notations to define Littlewood-Richardson coefficient in this section. More details can be found, for example, in [6] or [14]. By a partition, we mean a weakly decreasing sequence of nonnegative integers with finite number of positive integers. Let $\lambda=\left(\lambda_{1}, \lambda_{2}, \ldots\right)$ be a partition. The size of $\lambda$ is defined as $|\lambda|=\sum_{i} \lambda_{i}$, the total sum of the parts, and the length of $\lambda$, denoted by $\ell(\lambda)$, is defined as the number of positive integers in $\lambda$.

For each partition $\lambda$, we associate its Young diagram, a left-justified array of boxes with $\lambda_{p}$ boxes in its $p$ th row. We will let $(p, q)$-position of $\lambda$ denote the box at the $p$ th row and the $q$ th column in the Young diagram of $\lambda$. For a given partition $\lambda, \tilde{\lambda}$ indicates the conjugate of $\lambda$, whose diagram has $\lambda_{p}$ boxes in its $p$ th column. A Young tableau, or simply a tableau, of shape $\lambda$ is a filling of a Young diagram of shape

Received February 2, 2010. Accepted May 28, 2010.

2000 Mathematics Subject Classification: [2000] Primary 05E10; Secondary 14M15, 20C30.

Key words and phrases: Reduction formulae, Littlewood-Richardson coefficient, Schubert calculus.

This work was supported by the faculty research fund of Sejong University in 2007. 
$\lambda$ with integers that is weakly increasing across each row and strictly increasing down each column.

For two partitions $\lambda=\left(\lambda_{1}, \lambda_{2}, \ldots\right)$ and $\nu=\left(\nu_{1}, \nu_{2}, \ldots\right)$, we write $\lambda \subseteq \nu$, if $\lambda_{i} \leq \nu_{i}$ for all $i$. For partitions $\lambda$ and $\nu$ with $\lambda \subseteq \nu$, the skew diagram, or skew shape, $\nu / \lambda$ is the diagram consisting boxes of $\nu$ which are not the boxes of $\lambda$. We will write $(p, q) \in \nu / \lambda$ if $\lambda_{p}<q \leq \nu_{p}$, i.e there is a box at the $p$ th row and $q$ th column in the skew diagram of $\nu / \lambda$.

A skew tableau of shape $\nu / \lambda$ is a filling of boxes of a skew diagram $\nu / \lambda$ with integers, where entries are weakly increasing in rows and strictly increasing in columns. For a given (skew) tableau $T$ of shape $\nu / \lambda$ and $(p, q) \in \nu / \lambda$, we will let $T(p, q)$ denote the entry in the box at $(p, q)$ position of the tableau $T$. The content of a (skew) tableau $T$ is a sequence of integer $\mu=\left(\mu_{1}, \mu_{2}, \ldots\right)$ such that there are $\mu_{i} i$ 's in $T$. The reverse row word of a skew tableau $T$, denoted by $w(T)$, is the word obtained by reading the entries of $T$ from right to left and top to bottom starting from the first row. A word $w=x_{1} \cdots x_{r}$ is called a lattice word if, for any $s \leq r$ and $i, x_{1} \cdots x_{s}$ contains at least as many $i$ 's as it contains $(i+1)$ 's. A skew tableau $T$ is a Littlewood-Richardson skew tableau (LR-tableau) if its reverse row word $w(T)$ is a lattice word. If $T$ is an LR-tableau of shape $\nu / \lambda$, the lattice word condition enforce that the content $\mu$ of $T$ is a partition and $\mu \subseteq \nu$.

Definition 0.1. For given partitions $\lambda, \mu$ and $\nu$ with $\lambda, \mu \subseteq \nu$, the Littlewood-Richardson coefficient (LR-coefficient) $c_{\lambda, \mu}^{\nu}$ is the number of LR-tableaux on the shape $\nu / \lambda$ of content $\mu$.

The following is a well-known identity between LR-coefficients under conjugation.

Lemma $0.2([9,6])$. For partitions $\lambda, \mu, \nu$, the following equality holds:

$$
c_{\lambda, \mu}^{\nu}=c_{\tilde{\lambda}, \tilde{\mu}}^{\tilde{\nu}} .
$$

The Littlewood-Richardson coefficients occur in many mathematical subjects. They explain multiplication rule of the product of Schur functions in ring of symmetric functions. They also count the multiplicity in the decomposition of tensor products of irreducible polynomial representations of general linear groups, or in the decomposition of induced representations in the representation theory of the symmetric group. Moreover, the Littlewood-Richardson coefficients appear as intersection numbers in the Schubert calculus of Grassmannians.

For a given partition $\lambda$, by $\lambda \backslash \lambda_{I}$ where $I$ is a (finite) subset of the indices, we mean the partition obtained by deleting all the $i$ th part 
for $i \in I$ from $\lambda$. By a reduction formula for Littlewood-Richardson coefficient $c_{\lambda, \mu}^{\nu}$, is meant a formula of the type $c_{\lambda, \mu}^{\nu}=c_{\lambda^{\prime}, \mu^{\prime}}^{\nu^{\prime}}$, where either $\lambda^{\prime}=\lambda \backslash \lambda_{I}$ or $\lambda^{\prime}$ is the partition obtained by successively decreasing the first $i$ parts by 1 for $i \in I$, with similar formulae applying to the parts of $\mu$ and $\nu$ for $J$ and $K$ in which $J$ and $K$ necessary have the same cardinality as $I$. Note that the second type reduction formulae may obtained from the first type reduction formulae applying the identity under conjugation, Lemma 0.2 .

Inspired by the reduction formulae between intersection numbers on Grassmannians obtained by Griffiths-Harris [7] and the factorization theorem on Littlewood-Richardson coefficients due to King, Tollu and Toumazet $[10,11]$, the author, in collaboration with S. Cho and E.-K. Jung, has established eight such reduction formulae, $r=1, r=n-1$, $r=2$ and $r=n-2$ and their conjugates, where $n$ is an integer bigger than the length of $\nu$, in a sequence of previous papers $[1,3,2,4,5]$. A bijective proof, using LR-tableaux, of reduction formulae for $r=n-1$ and $r=n-2$ was announced in [1] and [4]. But detailed proofs for $r=1$ and $r=2$ reduction formulae have not been appeared in printed form. The purpose of this paper is to obtain a bijective proof of $r=1$ reduction formulae. In [3] and [5], only the statement and algorithm of bijective map for $r=1$ reduction formula appeared and no proof was given.

\section{Statements and Algorithm}

In this section, we give a precise statement of our $r=1$ reduction formula and describe a bijection which will be used to prove the reduction formula. Recall, for a given partition $\lambda$, by $\left.\lambda \backslash \lambda_{\{} i\right\}$ where $i$ is an index of the partition $\lambda$, we mean the partition obtained by deleting the $i$ th part from $\lambda$.

Theorem 1.1 ( $r=1$ reduction formula). We let $\lambda, \mu$ and $\nu$ be partitions with $\ell(\lambda), \ell(\mu), \ell(\nu) \leq n$ and $c_{\lambda, \mu}^{\nu}>0$. Suppose that there are $1 \leq i, j, k \leq n$ such that $i+j=k+1$ and $\nu_{k}=\lambda_{i}+\mu_{j}$. Then $c_{\lambda, \mu}^{\nu}=c_{\lambda \backslash \lambda_{\{i\}}, \mu \backslash \mu_{\{j\}}}^{\nu \backslash .}$.

For a skew tableau $T$ with $n$ rows and integers $1 \leq h, \ell \leq n$, we let $\mathfrak{n}_{T}^{h}(\ell)$ be the number of $\ell$ 's in the $h$ th row of $T$. We will need the following lemmas adopted from [1]. 
Lemma 1.2. A skew tableau $T$ with $n$ rows is an $L R$-tableau, i.e. the reverse row word $w(T)$ is a lattice word, if and only if, for all $1<p, s \leq n$,

$$
\sum_{h=1}^{p} \mathfrak{n}_{T}^{h}(s) \leq \sum_{h=1}^{p-1} \mathfrak{n}_{T}^{h}(s-1) .
$$

Lemma 1.3. Suppose that $s$ appears in the qth row of an LR-tableau $T$, then $q \geq s$.

In the rest of this paper, we assume that $i+j=k+1, \lambda_{i}+\mu_{j}=\nu_{k}$. The following is a key lemma that allows Definition 1.8 is valid.

Lemma 1.4. Let $T$ be an LR-tableau of shape $\nu / \lambda$ with content $\mu$. For $j+1 \leq \ell \leq k$, we have

$$
\begin{aligned}
\sum_{h=\ell}^{k} \mathfrak{n}_{T}^{h}(\ell) & =\sum_{h=\ell-1}^{k-1} \mathfrak{n}_{T}^{h}(\ell-1), \\
\sum_{h=j}^{k} \mathfrak{n}_{T}^{k}(h) & =\mu_{j},
\end{aligned}
$$

and

$$
\sum_{h=j}^{k} \mathfrak{n}_{T}^{h}(j)=\mu_{j}
$$

Proof. First note that the column (strict) increasingness of $T$ and Lemma 1.3 imply the entry in the $\left(k, \lambda_{i}+1\right)$-box is at least $k-i+1=j$. Then,

$$
\begin{aligned}
\mu_{j} & =\nu_{k}-\lambda_{i} \\
& \leq \mathfrak{n}_{T}^{k}(j)+\mathfrak{n}_{T}^{k}(j+1)+\cdots+\mathfrak{n}_{T}^{k}(k-2)+\mathfrak{n}_{T}^{k}(k-1)+\mathfrak{n}_{T}^{k}(k) \\
& \leq \mathfrak{n}_{T}^{k}(j)+\mathfrak{n}_{T}^{k}(j+1)+\cdots+\mathfrak{n}_{T}^{k}(k-2)+\left(\mathfrak{n}_{T}^{k}(k-1)+\mathfrak{n}_{T}^{k-1}(k-1)\right) \\
& \leq \mathfrak{n}_{T}^{k}(j)+\mathfrak{n}_{T}^{k}(j+1)+\cdots+\left(\mathfrak{n}_{T}^{k}(k-2)+\left(\mathfrak{n}_{T}^{k-1}(k-2)+\mathfrak{n}_{T}^{k-2}(k-2)\right)\right) \\
& \vdots \\
& \leq \mathfrak{n}_{T}^{k}(j)+\mathfrak{n}_{T}^{k-1}(j)+\cdots+\mathfrak{n}_{T}^{j}(j) \leq \mu_{j} .
\end{aligned}
$$

Therefore all inequalities are actually equalities and we obtain desired relations. 
Let $T$ be an LR-tableau of shape $\nu / \lambda$ with content $\mu$. The following algorithm is to construct the corresponding LR-tableau $\Phi(T)$ of shape $\left(\nu \backslash \nu_{\{k\}}\right) /\left(\lambda \backslash \lambda_{\{i\}}\right)$, with content $\mu \backslash \mu_{\{j\}}$ in a bijective way.

Definition 1.8. The reduced LR-tableau $\Phi(T)$ is obtained by applying the following algorithm:

Step 1: $\quad$ for $\ell=k$ downto $j+1$ do

Empty all boxes containing $\ell$ in $k$ th row.

Replace all $(\ell-1)$ 's in $(k-\ell+1)$ consecutive rows from the $(\ell-1)$ st row to the $(k-1)$ st row with $\ell$ 's.

end for

Step 2: $\quad$ Empty all boxes containing $j$ in the $k$ th row.

Step 3: $\quad$ for $\ell=j+1$ to $n$ do

Replace all $\ell$ 's with $(\ell-1)$ 's.

end for

Step 4: $\quad$ Slide each box at $(p, q)$-position, $1 \leq p \leq k-1, \lambda_{i}+1 \leq q$, one step down to remove all empty boxes.

Note (1.7) guarantees that we have removed all $j$ 's in $T$ after Step 2.

Example 1.9. Let $n=6$ and $\lambda=(5,5,3,2,2,0), \mu=(8,7,5,5,4,1)$, $\nu=(11,9,8,8,8,3)$. We have $i=3, j=3, k=5$, and $\lambda_{3}+\mu_{3}=\nu_{5}=8$.

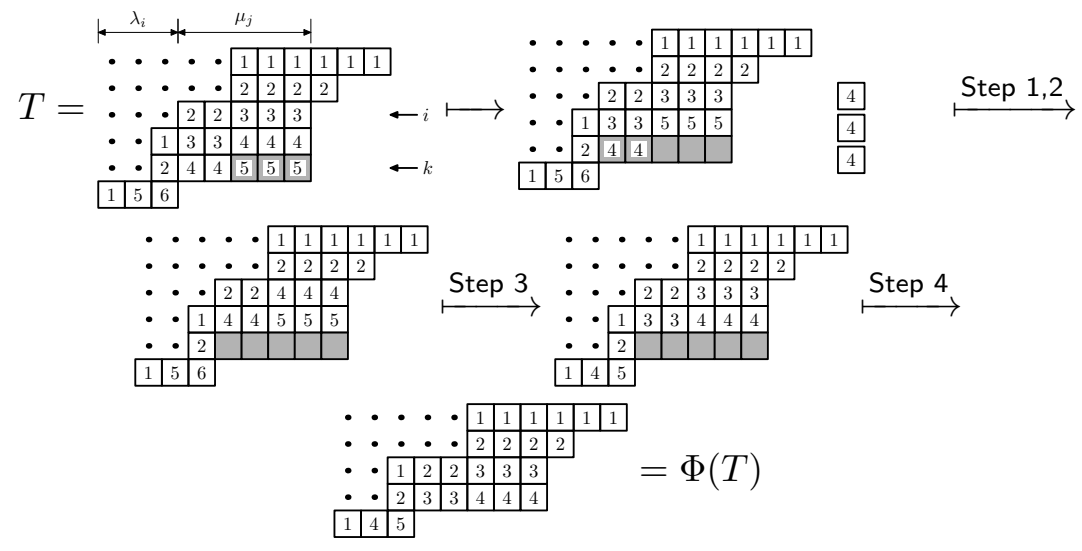

\section{Bijective Proof}

In this section, we will show that $\Phi$ is a bijective map between the set of LR-tableaux of shape $\nu / \lambda$ and content $\mu$ and the set of LR-tableaux of shape $\left(\nu \backslash \nu_{\{k\}}\right) /\left(\lambda \backslash \lambda_{\{i\}}\right)$ and content $\mu \backslash \mu_{\{j\}}$. For convenience, we denote the tableau obtained from $T$ by applying the Step 1 to Step 3 
by $T^{\circ}$, hence $T^{\circ}$ is a filling of boxes of a skew diagram $\nu / \lambda$ with empty boxes in the $k$ th row. The following two lemmas follow directly from Definition 1.8.

Lemma 2.1. For $(p, q) \in \nu / \lambda$, we have

$$
T^{\circ}(p, q)= \begin{cases}\text { empty box } & \text { if } p=k \text { and } q \geq \lambda_{i}+1, \\ T(p, q)-1, & \text { if } p>k \text { and } T(p, q) \geq j+1, \\ T(p, q), & \text { otherwise. }\end{cases}
$$

Lemma 2.2. For $(p, q) \in\left(\nu \backslash \nu_{\{k\}}\right) /\left(\lambda \backslash \lambda_{\{i\}}\right)$, we have

$$
\Phi(T)(p, q)= \begin{cases}T^{\circ}(p+1, q), & \text { if } i \leq p \leq k-1 \text { and } q \leq \lambda_{i}, \\ T^{\circ}(p, q), & \text { if } 1 \leq p \leq k-1 \text { and } q \geq \lambda_{i}+1, \\ T^{\circ}(p+1, q), & \text { if } p \geq k .\end{cases}
$$

Lemma 2.3. For $p$ with $i \leq p \leq k$, we have $T\left(p, \lambda_{i}+1\right) \geq p-i+1$. Also, if $\left(p, \lambda_{i}\right) \in \nu / \lambda$, then $T\left(p, \lambda_{i}\right) \leq p-i$ for $i<p \leq k$.

Proof. Note $\left(\ell, \lambda_{i}+1\right) \in \nu / \lambda$ for each $\ell=i, i+1, \ldots, p$. Thus, $T\left(p, \lambda_{i}+1\right) \geq p-i+1$ because of the column strictness of $T$.

Now note if $\left(p, \lambda_{i}\right) \in \nu / \lambda$ for $i<p \leq k$, then $\left(k, \lambda_{i}\right) \in \nu / \lambda$. Equation (1.6) and the condition $\lambda_{i}+\mu_{j}=\nu_{k}$ imply that $T\left(k, \lambda_{i}\right) \leq j-1$. Then, because of the column strictness of $T$ again, we have $T\left(p, \lambda_{i}\right) \leq$ $j-1-(k-p)=p-i$.

Proposition 2.4. $\Phi(T)$ is column increasing.

Proof. It is enough to show that $T^{\circ}$ is column increasing if we ignore empty boxes. And, from Lemma 2.1 and Lemma 2.3, it is clear that $T^{\circ}$ is column increasing.

Proposition 2.5. $\Phi(T)$ is weakly increasing in rows.

Proof. It is clear that rows of $T^{\circ}$ are weakly increasing if we ignore empty boxes from Lemma 2.1. From Lemma 2.1 and Lemma 2.2, we only need to compare $T^{\circ}\left(p, \lambda_{i}\right)$ and $T^{\circ}\left(p-1, \lambda_{i}+1\right)$ for $i<p \leq k$. Now Lemma 2.3 says that

$$
T^{\circ}\left(p, \lambda_{i}\right)=T\left(p, \lambda_{i}\right) \leq p-i \leq T\left(p-1, \lambda_{i}+1\right)=T^{\circ}\left(p-1, \lambda_{i}+1\right),
$$

and hence we have the row weakly increasingness of $\Phi(T)$.

Finally, we show that $\Phi(T)$ is an LR-tableau, i.e., the row word $w(\Phi(T))$ is a lattice word. We need the following lemma which immediately follows from Lemma 2.1 and Lemma 2.2. 
Lemma 2.6. For $1 \leq p, s \leq n-1$,

$$
\mathfrak{n}_{\Phi(T)}^{p}(s)= \begin{cases}\mathfrak{n}_{T}^{p}(s)+\mathfrak{n}_{T}^{p+1}(s) & \text { if } i \leq p \leq k-1 \text { and } s=p-i+1, \\ \mathfrak{n}_{T}^{p+1}(s) & \text { if } i \leq p \leq k-1 \text { and } s \leq p-i, \\ \mathfrak{n}_{T}^{p+1}(s) & \text { if } k \leq p \leq n-1 \text { and } s \leq j-1 \\ \mathfrak{n}_{T}^{p+1}(s+1) & \text { if } k \leq p \leq n-1 \text { and } s \geq j \\ \mathfrak{n}_{T}^{p}(s) & \text { otherwise. }\end{cases}
$$

Proposition 2.7. The reverse row word $w(\Phi(T))$ is a lattice word.

Proof. From Lemma 1.2, it is enough to show

$$
\sum_{h=1}^{p} \mathfrak{n}_{\Phi(T)}^{h}(s) \leq \sum_{h=1}^{p-1} \mathfrak{n}_{\Phi(T)}^{h}(s-1)
$$

for any $1<p, s \leq n-1$.

If $1<p \leq k-1$ and $s>p-i$, then

$$
\sum_{h=1}^{p} \mathfrak{n}_{\Phi(T)}^{h}(s)=\sum_{h=1}^{p} \mathfrak{n}_{T}^{h}(s) \leq \sum_{h=1}^{p-1} \mathfrak{n}_{T}^{h}(s-1)=\sum_{h=1}^{p-1} \mathfrak{n}_{\Phi(T)}^{h}(s-1) .
$$

If $i<p \leq k-1$ and $s \leq p-i$, then

$$
\begin{aligned}
& \sum_{h=1}^{p} \mathfrak{n}_{\Phi(T)}^{h}(s) \\
= & \sum_{h=s}^{s+i-2} \mathfrak{n}_{\Phi(T)}^{h}(s)+\mathfrak{n}_{\Phi(T)}^{s+i-1}(s)+\sum_{h=s+i}^{p} \mathfrak{n}_{\Phi(T)}^{h}(s) \\
= & \sum_{h=s}^{s+i-2} \mathfrak{n}_{T}^{h}(s)+\mathfrak{n}_{T}^{s+i-1}(s)+\mathfrak{n}_{T}^{s+i}(s)+\sum_{h=s+i}^{p} \mathfrak{n}_{T}^{h+1}(s) \quad \text { by Lemma } 2.6 \\
= & \sum_{h=1}^{p+1} \mathfrak{n}_{T}^{h}(s) .
\end{aligned}
$$

Similarly, we also have

$$
\sum_{h=1}^{p-1} \mathfrak{n}_{\Phi(T)}^{h}(s-1)=\sum_{h=1}^{p} \mathfrak{n}_{T}^{h}(s-1) .
$$

Hence, because $w(T)$ is a lattice word, we have

$$
\sum_{h=1}^{p} \mathfrak{n}_{\Phi(T)}^{h}(s)=\sum_{h=1}^{p+1} \mathfrak{n}_{T}^{h}(s) \leq \sum_{h=1}^{p} \mathfrak{n}_{T}^{h}(s-1)=\sum_{h=1}^{p-1} \mathfrak{n}_{\Phi(T)}^{h}(s-1) .
$$


If $p \geq k$ and $s \leq j-1$, then

$$
\sum_{h=1}^{p} \mathfrak{n}_{\Phi(T)}^{h}(s)=\sum_{h=1}^{p+1} \mathfrak{n}_{T}^{h}(s) \leq \sum_{h=1}^{p} \mathfrak{n}_{T}^{h}(s-1)=\sum_{h=1}^{p-1} \mathfrak{n}_{\Phi(T)}^{h}(s-1) .
$$

Now we note that, for $p \geq k$ and $s \geq j$,

$$
\begin{aligned}
\sum_{h=1}^{p} \mathfrak{n}_{\Phi(T)}^{h}(s) & =\sum_{h=1}^{k-1} \mathfrak{n}_{T}^{h}(s)+\sum_{h=k+1}^{p+1} \mathfrak{n}_{T}^{h}(s+1) \\
& =\sum_{h=1}^{k} \mathfrak{n}_{T}^{h}(s+1)+\sum_{h=k+1}^{p+1} \mathfrak{n}_{T}^{h}(s+1) \quad \text { by }(1.5) \\
& =\sum_{h=1}^{p+1} \mathfrak{n}_{T}^{h}(s+1) .
\end{aligned}
$$

If $p=k$ and $s=j$, then we have

$$
\begin{aligned}
\sum_{h=1}^{k} \mathfrak{n}_{\Phi(T)}^{h}(j) & =\sum_{h=1}^{k+1} \mathfrak{n}_{T}^{h}(j+1) \leq \sum_{h=1}^{k} \mathfrak{n}_{T}^{h}(j) \\
& \leq \sum_{h=1}^{k-1} \mathfrak{n}_{T}^{h}(j-1) \leq \sum_{h=1}^{k} \mathfrak{n}_{T}^{h}(j-1)=\sum_{h=1}^{k-1} \mathfrak{n}_{\Phi(T)}^{h}(j-1) .
\end{aligned}
$$

If $p=k$ and $s>j$, then

$\sum_{h=1}^{k} \mathfrak{n}_{\Phi(T)}^{h}(s)=\sum_{h=1}^{k+1} \mathfrak{n}_{T}^{h}(s+1) \leq \sum_{h=1}^{k} \mathfrak{n}_{T}^{h}(s) \leq \sum_{h=1}^{k-1} \mathfrak{n}_{T}^{h}(s-1)=\sum_{h=1}^{k-1} \mathfrak{n}_{\Phi(T)}^{h}(s-1)$.

If $p>k$ and $s>j$, then we have

$$
\sum_{h=1}^{p} \mathfrak{n}_{\Phi(T)}^{h}(s)=\sum_{h=1}^{p+1} \mathfrak{n}_{T}^{h}(s+1) \leq \sum_{h=1}^{p} \mathfrak{n}_{T}^{h}(s)=\sum_{h=1}^{p-1} \mathfrak{n}_{\Phi(T)}^{h}(s-1) .
$$

Now we show $\Phi$ is in fact a bijective map between the set of LRtableaux of shape $\nu / \lambda$ and content $\mu$ and the set of LR-tableaux of shape $\left(\nu \backslash \nu_{\{k\}}\right) /\left(\lambda \backslash \lambda_{\{i\}}\right)$ and content $\mu \backslash \mu_{\{j\}}$. Assume $T$ and $S$ are given LR-tableaux of shape $\nu / \lambda$ and content $\mu$ such that $\Phi(T)=\Phi(S)$. Lemma 2.1 and Lemma 2.2 imply that $T(p, q)=S(p, q)$ unless $p=k$ and $q \geq \lambda_{i}+1$. Then, because both $T$ and $S$ have content $\mu$ and rows are weakly increasing, there is a unique way to fill boxes at positions 
$(k, q)$ for $q=\lambda_{i}+1, \ldots, \nu_{k}$ for $T$ and $S$. Therefore, we obtain $T=S$, and hence $\Phi$ is an injective map.

Moreover, in general, $c_{\lambda, \mu}^{\nu} \geq c_{\lambda \backslash \lambda_{\{i\}}, \mu \backslash \mu_{\{j\}}}^{\nu \backslash \nu_{\{k\}}}$ for any $1 \leq i, j, k \leq n$ with $\lambda_{i}+\mu_{j}=\nu_{k}$ (see [8], and also [15]). Hence, we have the following theorem.

Theorem 2.9. The map $\Phi$ is a bijective map between the set of LR-tableaux of shape $\nu / \lambda$ and content $\mu$ and the set of LR-tableaux of shape $\left(\nu \backslash \nu_{\{k\}}\right) /\left(\lambda \backslash \lambda_{\{i\}}\right)$ and content $\mu \backslash \mu_{\{j\}}$.

\section{Application}

Theorem 1.1 is rather restrictive to be applied because we have to check the condition that $c_{\lambda, \mu}^{\nu}>0$ first. However, using the Horn's inequalities in $[12,13]$, we can enhance Theorem 1.1, and remove the condition $c_{\lambda, \mu}^{\nu}>0$. More detailed technique to obtain the extension from Theorem 1.1 can be found in [5].

Theorem 3.1 (Extension of Theorem 1.1). Let $\lambda, \mu$ and $\nu$ be partitions with $\ell(\lambda), \ell(\mu), \ell(\nu) \leq n$ and $|\nu|=|\lambda|+|\mu|$. Suppose that there are $1 \leq i, j, k \leq n$ satisfying the following condition;

$$
i+j \leq k+1 \text {. }
$$

(a) If $\lambda_{i}+\mu_{j}<\nu_{k}$, then $c_{\lambda, \mu}^{\nu}=0$.

(b) If $\lambda_{i}+\mu_{j}=\nu_{k}$, then $c_{\lambda, \mu}^{\nu}=c_{\lambda \backslash \lambda_{\{i\}}, \mu \backslash \mu_{\{j\}}}^{\nu \backslash \nu_{\{k\}}}$.

For a partition $\lambda$ and an integer $i \leq \ell(\lambda)$, we denote the integer sequence $\left(\lambda_{1}-1, \lambda_{2}-1, \ldots, \lambda_{i}-1, \lambda_{i+1}, \ldots\right)$ by $\lambda-\left(1^{i}\right)$. Note if $\lambda_{i}>\lambda_{i+1}$, then $\lambda-\left(1^{i}\right)$ is a partition obtained from $\lambda$ by removing a column of length $i$. A conjugated form of Theorem 3.1 can be obtained by applying Lemma 0.2. Again, one may find detailed proof to obtain the following theorem from [5]

Theorem 3.2 (Conjugation of Theorem 3.1). Let $\lambda, \mu$ and $\nu$ be partitions with $\ell(\lambda), \ell(\mu), \ell(\nu) \leq n$ and $|\nu|=|\lambda|+|\mu|$. Suppose that there are $\lambda_{i}, \mu_{j}, \nu_{k}$ such that $\lambda_{i}>\lambda_{i+1}, \mu_{j}>\mu_{j+1}$ and $\nu_{k}>\nu_{k+1}$ with $\lambda_{i+1}+\mu_{j+1}+1 \leq \nu_{k}$.

(a) If $i+j<k$, then $c_{\lambda, \mu}^{\nu}=0$.

(b) If $i+j=k$, then $c_{\lambda, \mu}^{\nu}=c_{\lambda-\left(1^{i}\right), \mu-\left(1^{j}\right)}^{\nu-\left(1^{k}\right)}$. 
Moreover, the formula can be applied by formally setting $\lambda_{0}=\mu_{0}=\nu_{1}$ and $\nu_{n+1}=0$.

Example 3.3. The following gives an example of applying Theorem 3.1 and Theorem 3.2 repeatedly to compute a Littlewood-Richardson coefficient. We use notations $\stackrel{1}{=}$ and $\stackrel{\widetilde{1}}{=}$ to indicate that Theorem 3.1 or Theorem 3.2 is used respectively.

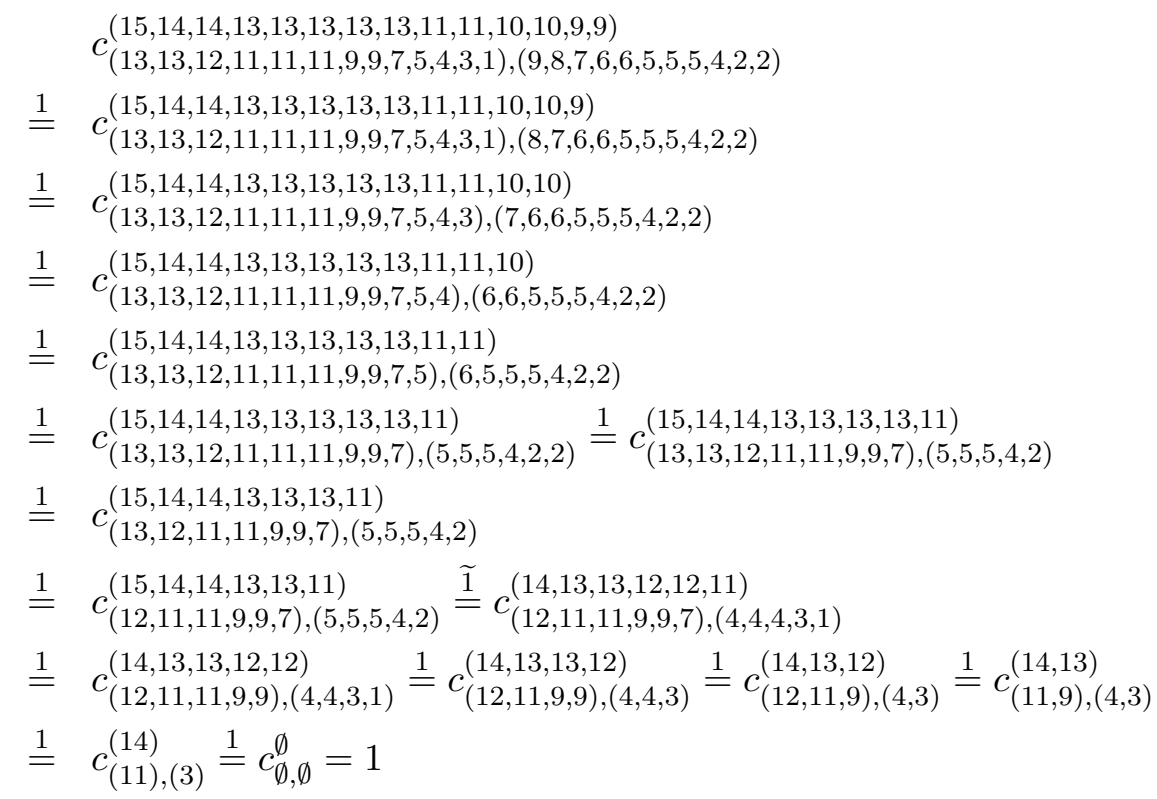

\section{References}

1. Soojin Cho, Eun-Kyoung Jung, and Dongho Moon, A combinatorial proof of the reduction formula for Littlewood-Richardson coefficients, J. Combin. Theory Ser. A 114 (2007), no. 7, 1199-1219.

2., A bijective proof of the second reduction formula for LittlewoodRichardson coefficients, Bull. Korean Math. Soc. 45 (2008), no. 3, 485-494.

3. - Reduction formulae from the factorization theorem of LittlewoodRichardson polynomials by King, Tollu and Toumazet, 20th International Conference on Formal Power Series and Algebraic Combinatorics, DMTCS Proceedings, vol. AJ, 2008, pp. 483-494.

4. __ An extension of reduction formula for littlewood-richardson coefficients, to appear in Journal of Korean Mathematical Society, 2010.

5. Soojin Cho and Dongho Moon, Reduction formulae of Littlewood-Richardson coefficients, to appear in Advances in Applied Mathematics, 2010.

6. William Fulton, Young tableaux, London Mathematical Society Student Texts, vol. 35, Cambridge University Press, Cambridge, 1997, With applications to representation theory and geometry. 
7. Phillip Griffiths and Joseph Harris, Principles of algebraic geometry, WileyInterscience [John Wiley \& Sons], New York, 1978.

8. Christian Gutschwager, On multiplicity-free skew characters and the Schubert calculus, arXiv:math/0608145v2, to appear in Ann. Comb., 2006.

9. Phil Hanlon and Sheila Sundaram, On a bijection between Littlewood-Richardson fillings of conjugate shape, J. Combin. Theory Ser. A 60 (1992), no. 1, 1-18.

10. R. C. King, C. Tollu, and F. Toumazet, The hive model and the polynomial nature of stretched Littlewood-Richardson coefficients, Séminaire Lotharingien de Combinatoire 54A (2006), 1-19.

11. , J. Combin. Theory Ser. A 116 (2009), no. 2, 314-333.

12. Alexander A. Klyachko, Stable bundles, representation theory and Hermitian operators, Selecta Math. (N.S.) 4 (1998), no. 3, 419-445.

13. Allen Knutson, Terence Tao, and Christopher Woodward, The honeycomb model of $\mathrm{GL}_{n}(\mathbb{C})$ tensor products. II. Puzzles determine facets of the LittlewoodRichardson cone, J. Amer. Math. Soc. 17 (2004), no. 1, 19-48 (electronic).

14. Bruce E. Sagan, The symmetric group, second ed., Graduate Texts in Mathematics, vol. 203, Springer-Verlag, New York, 2001, Representations, combinatorial algorithms, and symmetric functions.

15. John R. Stembridge, Multiplicity-free products of Schur functions, Ann. Comb. 5 (2001), no. 2, 113-121.

Department of Applied Mathematics

Sejong University

Seoul 143-747, Korea

E-mail: dhmoon@sejong.ac.kr 